\title{
Bioprospecting - Gerechte Verteilung genetischer Ressourcen
}

In: Nida-Rümelin, Julian / Daniels, Detlef von / Wloka, Nicole (Hrsg.): Internationale Gerechtigkeit und institutionelle Verantwortung. - ISBN: 978-3-11-061586-9. - Berlin: De Gruyter, 2019.

S. 341-355

(Forschungsberichte / Interdisziplinäre Arbeitsgruppen, Berlin-Brandenburgische Akademie der Wissenschaften ; 41) 


\section{Bioprospecting - Gerechte Verteilung genetischer Ressourcen}

\section{Einführung}

Gerechtigkeitsfragen, die neu beantwortet werden müssen, weil Forschung und Technik voranschreiten, stellen sich in vielen Bereichen unseres Lebens. Besonders offensichtlich und umstritten ist dies für die Biotechnologie, die Genomeditierung und die künstliche Intelligenz (vgl. zu Fragen der Regulierung von Wissenschaft und Technik aus menschenrechtlicher Sicht auch Vöneky 2018). Doch auch in den weniger öffentlich diskutierten Gebieten stehen wir vor Anpassungs-, Legitimations- und Gerechtigkeitsproblemen, wenn neue Technologien entwickelt und eingesetzt werden. In den hier folgenden Ausführungen möchte ich den Schwerpunkt auf die Möglichkeit der Erkundung mariner genetischer Ressourcen und das sog. Bioprospecting legen. Gezeigt werden soll, welche Normen des internationalen Rechts, insbesondere des Seevölkerrechts, Bioprospecting heute bereits erfassen und warum einige Staaten der internationalen Gemeinschaft gerade diesen Bereich mit neuen und spezifischen seevölkerrechtlichen Normen regulieren möchten - auch wenn andere die pessimistische These vertreten, dass in einer Zeit der Unordnung Einigkeit über neue internationale Normen nicht erreicht werden kann.

Der Begriff Bioprospecting steht hier für Biological Diversity Prospecting, d.h. für die Erkundung kommerziell wertvoller genetischer Ressourcen. In der Regel geht es beim Bioprospecting darum, systematisch genetisches Material und darin enthaltene Informationen zu erkunden und zu sammeln sowie dieses später für die kommerzielle Nutzung durch Unternehmen, bspw. in der Pharma- oder Kosmetikindustrie, aufzubereiten (Mossop 2015, S. $825 \mathrm{ff}$.). Zum Teil werden vier Phasen unterschieden, die Bioprospecting wie folgt unterteilen: (1) das Erheben von Proben vor Ort, (2) die Isolation, Charakterisierung und Kultivierung spezifischer Verbindungen, (3) das Screening für mögliche Nutzungen und (4) die Entwicklung neuer Produkte (vgl. Leary 2010, S. 353 ff.).

Ein Indiz für das wirtschaftliche Potenzial dieser Art der Erkundung genetischer Ressourcen mag sein, dass heute die Hälfte aller Medikamente direkt oder indirekt aus in der Natur vorkommenden Substanzen gewonnen wird (Overmann/ Smith 2017, S. 59). Besonders attraktiv könnten genetische Ressourcen aus Tiefseemeeresgebieten sein (Vöneky/Beck 2017, Rdn 12). Besonders widerstandsfähige Organismen leben dort in bis zu 11.000 Metern Tiefe. Da diese sich an Druck

Ә OpenAccess. (C) 2019 Silja Vöneky, publiziert von De Gruyter. (cc) BY-NC-SA Dieses Werk ist lizenziert unter der Creative Commons Attribution-NonCommercial-ShareAlike 4.0 Lizenz. 
und Kälte angepasst haben, hoffen manche, dass aus ihnen neuartige Medikamente zur Behandlung bisher unheilbarer Krankheiten entwickelt werden können (Arico/Salpin 2005, S. 25 ff.). Im Folgenden soll auf die dafür zu erkundenden marinen genetischen Ressourcen eingegangenen werden, deren Produkte sog. Marine Genetic Ressources-Produkte (MGR-Products) sind (allein im Pharmasektor wird der Gesamtmarktwert dieser Produkte schon für das Jahr 2006 auf 643 Milliarden US-Dollar beziffert, s. Annweiler 2017, S. 33). Auch Naturwissenschaftler sehen im marinen Bioprospecting enormes Potenzial, warnen aber zugleich vor den drohenden Auswirkungen der wirtschaftlichen Ausbeutung der Tiefsee. Zur Bewahrung des ökologischen Gleichgewichts und damit auch zur langfristigen Erhaltung dort lebender Organismen müsse das Sammeln genetischen Materials systematisch und schonend durchgeführt werden (Synnes 2007, S. 57). Gefahren für die marinen genetischen Ressourcen werden daher auch im Bioprospecting und nicht nur im Tiefseebergbau und der Tiefseefischerei gesehen (Boetius/Haeckel 2018, S. 35). Es wird außerdem darauf hingewiesen, dass die bisher fehlenden Vereinbarungen zur Aufteilung der Vorteile Bioprospecting in der Zukunft einschränken könnten (Snelgrove 2016, S. 796f.). Ein rechtlicher Rahmen für Bioprospecting-Aktivitäten wird daher auch insoweit befürwortet (s. etwa Abida et al. 2013, S. 4604; Synnes 2007, S. 57).

Auch wenn Bioprospecting eine wirtschaftlich lohnende Technik des 21. Jahrhunderts sein könnte, entstammt das in diesem Bereich besonders relevante Seevölkerrecht dem letzten Jahrhundert. Das von mehr als 160 Staaten, darunter auch Deutschland, ratifizierte Seerechtsübereinkommen der Vereinten Nationen (SRÜ; United Nations Convention of the Law of the Sea, UNCLOS) ist 1994 in Kraft getreten und wurde bereits 1982 vereinbart. Bioprospecting hat sich erst seit den 1990er Jahren entwickelt. Es gibt daher auch in dem zum Teil besonders ausdifferenzierten Übereinkommen mit seinen 320 Artikeln keine Normen, die speziell oder ausdrücklich diese technische Anwendung regeln. Zudem sind Staaten, deren Unternehmen wichtige Vorreiter dieser Technik sind, z. B. die USA, keine Vertragspartei des Seerechtsübereinkommens. Für diese Staaten gelten lediglich die Genfer Seerechtsübereinkommen von 1958 und das Völkergewohnheitsrecht. Es stellt sich damit auch völkerrechtlich die Frage, ob die bestehenden Normen auf das Bioprospecting anwendbar und zur rechtlichen Einhegung geeignet und ausreichend sind. Insbesondere sind Vorteile, aber auch Lücken und Defizite der gegenwärtigen Normen zu untersuchen sowie Möglichkeiten zu deren sinnvoller und legitimer Fortentwicklung.

Die folgende Untersuchung soll sich dabei im Wesentlichen darauf beschränken, die differenzierten seevölkerrechtlichen Normen zu untersuchen, um die zentralen normativen Aspekte des Völkerrechts, wie es heute zu beachten ist, zu beleuchten. Weitere (völker-)rechtliche Fragen, insbesondere solche des Pa- 
tentrechts oder des Umweltvölkerrechts, die beispielsweise im Zusammenhang mit dem Übereinkommen über biologische Vielfalt (Biodiversitätskonvention; Convention on Biological Diversity, CBD) und dessen Protokollen auftreten, finden aus Platzgründen keine oder nur knappe Berücksichtigung. ${ }^{1}$ Ausgangspunkt ist vielmehr die Frage, welche Regeln nach dem geltenden Seevölkerrecht auf Bioprospecting anwendbar sind. Es wird sich zeigen, dass das Ergebnis, das aus der Anwendung der internationalen Normen folgt, Gerechtigkeitsfragen aufwirft, die das Seevölkerrecht der Gegenwart selbst nicht beantworten kann.

\section{Bioprospecting im Seevölkerrecht der Gegenwart}

Hinsichtlich der normativen Ausgestaltung nach dem internationalen Seerecht sind Gebiete innerhalb küstenstaatlicher Jurisdiktion von solchen außerhalb dieser Jurisdiktion zu unterscheiden. Dies ist wichtig, da es beim marinen Bioprospecting insbesondere auch, wie oben ausgeführt, um die Bewirtschaftung genetischer Ressourcen des Meeres in den Gebieten jenseits der Grenzen nationaler Hoheitsgewalt geht, die ca. 40 Prozent der globalen Meeresfläche umfassen.

$\mathrm{Zu}$ den Gebieten innerhalb küstenstaatlicher Jurisdiktion zählt seevölkerrechtlich das Küstenmeer, das sich bis maximal zwölf Seemeilen von den sog. Basislinien erstreckt (Art. 3 SRÜ). Der Souveränität des Küstenstaates unterliegen hierbei das Meer, der Meeresboden und der Meeresuntergrund einschließlich der dort befindlichen genetischen Ressourcen (Wolf 2012, Rdn. 10). Zu den Gebieten mit küstenstaatlichen Hoheitsbefugnissen zählen auch die ausschließliche Wirtschaftszone (AWZ) eines Staates, die sich nach Art. 57 SRÜ bis zu 200 Seemeilen von der Basislinie erstrecken darf, sowie der Festlandsockel. Diesen bilden der jenseits des Küstenmeeres gelegene Meeresboden und der Meeresuntergrund der Unterwassergebiete, die sich über die gesamte natürliche Verlängerung des Landgebietes des jeweiligen Staates bis zur äußeren Kante des Festlandrandes erstrecken. Der Festlandsockel ist begrenzt auf 200 Seemeilen mit Erweiterungsmöglichkeit auf 350 Seemeilen (Art. 76 Abs. 8 SRÜ).

Entscheidend ist, dass sich diese genannten Bereiche von denen jenseits der Grenzen nationaler Hoheitsgewalt unterscheiden (,areas beyond national jurisdiction"). Zu den Bereichen jenseits der Grenzen nationaler Hoheitsgewalt gehören die Hohe See und das sog. Gebiet (The Area). Das Gebiet wird in Art. 1 SRÜ

1 Der Begriff Bioprospecting ist jedoch ursprünglich weniger mit dem Seevölkerrecht verbunden als vielmehr mit der Biodiversitätskonvention von 1992 und deren Nagoya Protocol von 2010. 
als der Meeresboden und Meeresuntergrund jenseits der Grenzen des Bereichs nationaler Hoheitsbefugnisse definiert.

\section{A Bioprospecting in der ausschließlichen Wirtschaftszone}

Auch das Bioprospecting in der ausschließlichen Wirtschaftszone eines Staates wird durch Art. 56 Abs. 1 lit. a SRÜ eindeutig geregelt. Hiernach haben die Küstenstaaten auch in der ausschließlichen Wirtschaftszone souveräne Rechte zur Erforschung, Ausbeutung und Bewirtschaftung der lebenden natürlichen Ressourcen („living natural resources“) der Gewässer über dem Meeresboden, des Meeresbodens und seines Untergrunds (dazu näher Art. 56 Abs. 3 und 74 Abs. 4 SRÜ). Da die historische Auslegung eines völkerrechtlichen Vertrages wie des Seerechtsübereinkommens nur ergänzend heranzuziehen ist (Art. 32 des Wiener Übereinkommen über das Recht der Verträge, WVK), ist es nicht entscheidend, dass Bioprospecting bei den Vertragsverhandlungen unbekannt war. Die marinen genetischen Ressourcen des Bioprospecting sind unter den Wortlaut der natürlichen lebenden Ressourcen zu fassen, die in Art. 56 SRÜ genannt werden (so auch Proelß 2017, Rdn. 14; Mossop 2015, S. 825, 833) und auch in der Rechtsprechung des Internationalen Seegerichtshofes bestätigt wurden. ${ }^{2}$

Umstritten ist die Frage, ob Bioprospecting wissenschaftliche Forschung ist und damit (auch) unter Teil 13 (XIII) des Seerechtsübereinkommens fällt. Dies ist eine entscheidende Frage, auf die weiter unten einzugehen sein wird. Selbst wenn man sie jedoch bejahen würde, gilt in der ausschließlichen Wirtschaftszone, dass die Küstenstaaten das Recht haben, ihre Zustimmung zur Durchführung des Vorhabens durch einen anderen Staat zu versagen, wenn das Vorhaben - wie beim Bioprospecting - von unmittelbarer Bedeutung für die Erforschung und Ausbeutung der lebenden Ressourcen ist (Art. 246 Abs. 5 SRÜ) (s. zudem Matz 2002, S. 288).

Die Rechte der Küstenstaaten sind dabei nicht unbegrenzt: Grenzen sind insbesondere die Umweltschutzpflichten nach Art. 192ff. SRÜ. Dazu gehören die Pflicht zum Schutz und zur Bewahrung der Meeresumwelt (Art.192 SRÜ) sowie die Pflicht, Maßnahmen zur Verhütung, Verringerung und Überwachung der Verschmutzung der Meeresumwelt zu ergreifen (Art. 194-196 SRÜ). Daneben bestehen auch Pflichten, notwendige Maßnahmen zu ergreifen, um Emissionen zu reduzieren. Dies gilt ausdrücklich auch hinsichtlich der Maschinen, die eingesetzt

2 Vgl. ITLOS, Southern Bluefin Tuna Cases (New Zealand v Japan; Australia v Japan) (Provisional Measures) ITLOS Cases Nos 3, 4 vom 27. August 1999. 
werden, um natürliche Ressourcen zu erforschen oder auszubeuten, d.h. Bioprospecting durchzuführen (Art. 194 Abs. 3 lit. c SRÜ) (so auch Leroux/Mbengue 2010, S. 4). Umfasst ist ferner die Pflicht, die Einführung fremder und neuer Arten zu verhindern (Art. 196 SRÜ).

Diese seevölkerrechtlichen Pflichten zum Schutz der Umwelt werden umweltvölkerrechtlich ergänzt durch Pflichten aus der Biodiversitätskonvention, denn diese findet Anwendung in Gebieten, die staatlicher Hoheitsgewalt unterliegen. ${ }^{3}$ Staaten sind hiernach zur Überwachung der schädlichen Umwelteffekte von Aktivitäten verpflichtet, die unter ihrer Kontrolle vorgenommen werden (Art. 7 lit. c CBD). Auch ist ein Access and Benefit Sharing-Regime (ABS-Regime) in Kraft, das den Zugang anderer Staaten zu genetischen Ressourcen im Hoheitsgebiet eines Vertragsstaates sowie die gerechte und ausgewogene Verteilung von aus diesen Ressourcen gezogenen Vorteilen vorsieht (Art. 15 CBD und das konkretisierende Nagoya Protocol on Access and Benefit Sharing). Allerdings sind nicht alle Vertragsstaaten der Biodiversitätskonvention auch Vertragspartei des Nagoya-Protokolls. Relevante Staaten, wie u. a. Australien, Kanada oder Russland, sind an diese Regelungen nicht gebunden. Innerhalb dieser völkerrechtlichen Grenzen haben jedoch Küstenstaaten das Recht, selbst Bioprospecting durchzuführen oder durch öffentliche oder private Unternehmen durchführen zu lassen.

\section{B Bioprospecting auf dem Festlandsockel eines Staates}

Eine ähnliche völkerrechtliche Bewertung ergibt sich hinsichtlich des Bioprospecting auf dem Festlandsockel eines Staates. Nach Art. 77 Abs. 1 SRÜ dürfen natürliche Ressourcen („natural resources“) ausgebeutet werden, wobei darunter - im Gegensatz zu Art. 56 Abs. 1 lit. a SRÜ - nach Art. 77 Abs. 4 SRÜ nur zu sesshaften Arten gehörende Lebewesen (,living organisms belonging to sedentary species“) fallen. Hierzu zählen nach der Legaldefinition in Art. 77 SRÜ solche, die unbeweglich auf oder unter dem Meeresboden verbleiben oder sich nur in ständigem körperlichem Kontakt mit dem Meeresboden oder seinem Untergrund fortbewegen können. Bioprospecting-Aktivitäten sind daher beschränkt auf die genetischen Ressourcen der genannten sesshaften Arten auf dem Festlandsockel zulässig.

3 Außerdem findet die Biodiversitätskonvention bei allen Aktivitäten unter der Jurisdiktion und Kontrolle (,jurisdiction or control“) eines Vertragsstaates Anwendung, auch wenn diese in Gebieten jenseits staatlicher Hoheitsgewalt vorgenommen werden, vgl. Art. 4 CBD. 
Daraus folgt aber auch, dass Bioprospecting von den Küstenstaaten nach dieser Bestimmung nicht in der Wassersäule über dem Festlandsockel durchgeführt werden darf. Zum Teil wird diese Differenzierung als unplausibel kritisiert (Leroux/Mbengue 2010, S. 5). Das Seevölkerrecht des Seerechtsübereinkommens ist diesbezüglich aber gerade schon nach seinem Wortlaut eindeutig, auch in Bezug auf die neue Technik. Dies gilt, auch wenn manche Küstenstaaten gerne erweiterte Befugnisse über ihrem Festlandsockel in Anspruch nehmen würden.

Für Bioprospecting-Aktivitäten in Bezug auf sesshafte Arten auf dem Festlandsockel selbst ergeben sich Grenzen für die Küstenstaaten wieder durch Pflichten zum Schutz der Umwelt und zur Verhinderung von Verschmutzungen (Art. 192, 194-196, 204-206 SRÜ) sowie durch die Regeln der Biodiversitätskonvention. Innerhalb dieser Grenzen besteht jedoch auch in diesem Bereich für Küstenstaaten das Recht, selbst Bioprospecting durchzuführen oder durch Unternehmen durchführen zu lassen. Für die Wassersäule über dem Festlandsockel sowie für die nicht von Art. 77 Abs. 4 SRÜ erfassten nicht-sesshaften Arten gelten dagegen die Regelungen $\mathrm{zu}$ Gebieten jenseits nationaler Jurisdiktion (Mossop 2010, S. 324).

\section{Bioprospecting in den Gebieten jenseits nationaler Jurisdiktion}

Die größten seevölkerrechtlichen Probleme ergeben sich in Bezug auf Bioprospecting in den Gebieten jenseits nationaler Jurisdiktion, also jenseits der ausschließlichen Wirtschaftszone und jenseits des Festlandsockels eines Staates. Zunächst soll als Teil dieses Bereichs das Gebiet der Hohen See betrachtet werden.

Grundlegend statuiert Art. 87 Abs. 1 SRÜ die Freiheit der Hohen See für alle Staaten und nennt sechs Anwendungsbereiche, u.a. die Freiheit der Schifffahrt, des Überflugs, der Fischerei und der wissenschaftlichen Forschung. An dieser Stelle werden weder Bioprospecting noch die Erforschung und die Ausbeutung genetischer Ressourcen ausdrücklich erwähnt, was nicht erstaunlich ist, wenn man die zeitliche Genese des SRÜ einerseits und die spätere Entwicklung des Bioprospecting andererseits betrachtet.

Dennoch wird im Ergebnis zum Teil die Ansicht vertreten, dass Bioprospecting unter die Freiheit der Hohen See falle (Proelß 2007, S. 654). Als Argument führen die betreffenden Autoren an, dass Bioprospecting von der Freiheit der ausdrücklich genannten wissenschaftlichen Forschung (,freedom of scientific research“) erfasst werde (Art. 87 Abs. 1 lit. f SRÜ) (s. Proelß 2007, S. 654). Dieses bereits genannte - Problem, wie wissenschaftliche Forschung zu bestimmen ist, ist nicht einfach zu lösen, da im Seerechtsübereinkommen dieser Begriff nicht 
definiert ist (Vöneky/Beck 2017, Rdn 5, 7-9). Gegen eine Einordnung des Bioprospecting unter den Begriff der wissenschaftlichen Forschung ist jedoch einzuwenden, dass sich Wissenschaft und Bioprospecting in der Verwendung des gewonnenen Wissens und der Ergebnisse wesentlich voneinander unterscheiden (vgl. auch Report of the Secretary-General, Oceans and the Law of the Sea, UN Doc. A/59/62, Rdn 261). Zwar verändert nicht jede Gewinnabsicht oder jede Gewinnaussicht automatisch das Wesen der Tätigkeit von Meeresforschung unter dem Regime des Seerechtsübereinkommens (vgl. dazu und für das Folgende Vöneky/ Beck 2017, Rdn 12). Der entscheidende Faktor für die Einstufung einer Tätigkeit als wissenschaftliche Meeresforschung scheint jedoch sinnvoller Weise ihr hauptsächlicher oder übergeordneter Zweck zu sein. Wenn der Hauptzweck nicht mehr in der Mehrung menschlichen Wissens liegt, verändert die Feststellung kommerziell nutzbarer Informationen den Charakter der Tätigkeit. Bioprospecting verfolgt hauptsächlich praktische kommerzielle Zwecke. Solange das übergeordnete Ziel von Bioprospecting keinen ernsthaften und systematischen Versuch darstellt, Wissen zu erlangen, erweist sich Bioprospecting daher gerade nicht als wissenschaftliche Meeresforschung im Sinne des Seerechtsübereinkommens, die einem gesonderten Regime unterliegt und für die gelten muss, dass sie auch zum Wohle der gesamten Menschheit ausgeführt werden kann (Vöneky/Beck 2017, Rdn 12).

Überzeugender erscheint daher die Ansicht derjenigen, die vertreten, dass die Freiheit, Bioprospecting auf Hoher See durchzuführen, von den allgemeinen Regeln abgeleitet werden könne, also von dem Grundsatz der Freiheit der Hohen See nach Art. 87 SRÜ, da die Auflistung der besonderen Freiheiten in Art. 87 Abs. 1 lit. a-f SRÜ ausdrücklich nicht abschließend sei, wie der Wortlaut zeige (Leroux/ Mbengue 2010, S. 6). Im Sinne einer dynamischen Auslegung würde die Freiheit der Hohen See auch andere Nutzungen der Hohen See umfassen, gerade auch diejenigen, die nicht zur Zeit der Vertragsverhandlungen vorausgesehen wurden.

Dagegen wird jedoch eingewandt, dass die Freiheit der Hohen See auf die negativ abgegrenzte Wassersäule begrenzt bleibe (siehe Art. 86 S. 1 SRÜ). Sie beziehe sich nicht auf die lebenden Ressourcen des Meeresbodens (Annweiler 2017, S. 91). Das Gebietsregime erfasse zwar nur die mineralischen Ressourcen, aber das Regime der Hohen See betreffe dennoch gerade nicht die lebenden Ressourcen des Meeresbodens, vielmehr bestehe eine Lücke im vertraglichen System (Annweiler 2017, S. 91).

Nach anderer Ansicht spricht für eine Erstreckung jedoch, dass die Erhaltung und Bewirtschaftung der lebenden Ressourcen der Hohen See auch andere Lebewesen als Fische erfasst. Andernfalls hätten die sesshaften Arten nicht ausdrücklich von dem Regime der ausschließlichen Wirtschaftszone ausgenommen werden müssen (wie in Art. 68 SRÜ) (s. Proelß 2007, S. 654). Teil 7 (VII) des See- 
rechtsübereinkommens finde immer jenseits der ausschließlichen Wirtschaftszone bzw. des Küstenmeeres Anwendung; dieser Teil trete nur zurück, soweit er von den Bestimmungen bzgl. des Tiefseebodenbergbaus verdrängt werde (so auch Proelß 2007, S. 653).

Dies ist überzeugend, so dass Bioprospecting unter die Freiheiten der Hohen See fällt und daher einzelstaatlichen Zugangs- und Nutzungsbefugnissen unterliegt. Zugang, Nutzung und Management (Verteilung) genetischer Ressourcen sind damit jedoch gerade nicht durch das Seerechtsübereinkommen internationalisiert, sondern in das Ermessen der Staaten gestellt. Die nationalen Regime finden damit Anwendung für die Staatsangehörigen und -zugehörigen der jeweiligen Staaten, also natürliche Personen und Unternehmen, sowie auf die Aktivitäten, die von Schiffen vorgenommen werden, die unter der Flagge des jeweiligen Staates fahren.

Grenzen für Staaten ergeben sich allerdings wieder aus den generellen Pflichten zum Schutz der Umwelt (Art. 192 SRÜ) und zur Verschmutzungsverhütung (Art. 194-196 und 204-206 SRÜ) (vgl. dazu und für das Folgende bereits Leroux/Mbengue 2010). Da marines Bioprospecting der Tiefsee schaden kann (insbesondere Tiefseehabitaten wie den Tiefseehydrothermalquellen), wird richtigerweise vertreten, dass Staaten verpflichtet sind, einen Rechtsrahmen zu entwickeln, der es ermöglicht, die Schäden an der marinen Umwelt einzuschätzen, die durch solche Aktivitäten verursacht werden können. Zudem ist entscheidend, dass die Staaten sicherstellen, dass Bioprospecting, das unter ihrer Kontrolle durchgeführt wird, nicht mit anderen legitimen Nutzungen der Hohen See in Konflikt gerät. Staaten müssen daher Bioprospecting-Aktivitäten so regulieren, dass Meeresforschungsaktivitäten und Bergbau in dem Gebiet nicht ungerechtfertigt beeinträchtigt werden (Art. 240 lit. c, 147 Abs. 3 SRÜ).

Sonstige Regeln bezüglich des Zugangs und der Verteilung gibt es nach dem Seerechtsübereinkommen und Seevölkergewohnheitsrecht nicht, wenn es sich um genetische Ressourcen jenseits nationaler Hoheitsgewalt handelt. Vorteile der Nutzung (,benefits“) müssen gerade nicht mit anderen Staaten geteilt werden.

Keine relevante Ausnahme sind auch kommerzielle Nutzungen, die sich aus wissenschaftlichen Untersuchungen entwickelt haben: Zwar darf nach Art. 241 SRÜ keine Aneignung von Teilen der marinen Umwelt und ihrer Ressourcen (,any part of the marine environment and its resources") basierend auf Forschungsaktivitäten erfolgen. Allerdings sind nach überzeugender Ansicht gerade im Hinblick auf die Entstehungsgeschichte des Übereinkommens Eigentumsrechte von Art. 241 SRÜ ausgenommen (Matz-Lück 2017, Rdn 15). Zudem findet sich auch keine hinreichende Praxis der Vertragsstaaten, die eine weite Auslegung stützen würde. Schließlich sind diese Rechte auch keine direkte Konsequenz der For- 
schung, so dass sie vom Anwendungsbereich ausgenommen sind (Matz-Lück 2017, Rdn 16).

Auch müssen Ergebnisse mariner Forschung nach Art. 244 SRÜ publiziert und verbreitet werden, während die Ergebnisse von Bioprospecting vertraulich bleiben dürfen und wohl auch vertraulich sein müssen, wenn es wirtschaftlich sinnvoll sein soll. Das Problem sind hier jedoch mit dem Tiefsee-Bioprospecting einhergehende Gemeinschaftsaktivitäten, die oft wegen der hohen Kosten und Risiken notwendig sind. Hier ist unklar, ob Proben, die gesammelt werden, als Ergebnisse von mariner Forschung oder vielmehr von kommerziellem Bioprospecting gelten sollen.

Zusammenfassend lässt sich damit sagen, dass die Regelung von Bioprospecting in der Jurisdiktion der Staaten liegt, wenn auch mit den genannten Grenzen. Sie sind verantwortlich dafür, Regeln zu erlassen und durchzusetzen. Nach Art. 118 SRÜ müssen Staaten allerdings miteinander kooperieren (,States shall cooperate ..."). Auch wenn der eigentliche Anwendungsbereich von Art. 118 SRÜ Fische sind, gilt er für alle lebenden Ressourcen, einschließlich mariner genetischer Ressourcen (Rayfuse 2017, Rdn 18).

Nicht anwendbar ist jedoch die Biodiversitätskonvention: Es besteht keine Pflicht, genetische Ressourcen zu identifizieren und $\mathrm{zu}$ überwachen; auch das Access and Benefit Sharing-Regime gilt hier in Bezug auf die marinen genetischen Ressourcen des Tiefseebodens nicht. Dies ergibt sich sowohl aus der grundsätzlichen Beschränkung des Anwendungsbereichs der Biodiversitätskonvention auf Gebiete innerhalb nationaler Hoheitsgewalt (Art. 4 lit. a CBD) als auch aus dem Wortlaut des Art. 15 Abs. 1 CBD (,the sovereign rights of States over their natural resources“). Anwendung findet das Regime aber hinsichtlich der verwendeten Verfahren und Technologien (Art. 16 CBD) (s. insgesamt Matz 2002, S. 28, 292; Annweiler 2017, S. 107; Proelß 2008, S. 422).

Daraus ergibt sich, dass die Verantwortung für die Bewahrung und nachhaltige Nutzung der marinen genetischen Ressourcen in den Gebieten jenseits nationaler Jurisdiktion bei den Einzelstaaten liegt und nur wenigen völkerrechtlichen Grenzen unterliegt. Dies ist jedoch der Hauptkritikpunkt am gegenwärtigen Regime: In der Praxis gibt es nur wenige Staaten und Unternehmen, welche die Möglichkeit besitzen, das kostenintensive und technisch anspruchsvolle TiefseeBioprospecting durchzuführen. Daher nützt die aktuelle Rechtslage diesen wenigen Staaten. 


\section{Gerechtigkeitsfragen des Bioprospecting}

Welche Lösungen sind denkbar, will man dieses Ergebnis, das unbillig und ungerecht erscheint, vermeiden? Ein Ausweg wäre, dass alle Rechte an den genetischen Ressourcen jenseits nationaler Jurisdiktion der gesamten Menschheit zustehen. Dies wäre ganz unproblematisch bereits heute der Fall, wenn auf Bioprospecting die Regeln für das sog. Gebiet (also Meeresboden und Meeresuntergrund jenseits der Grenzen nationaler Hoheitsbefugnisse) nach den Art. 133 ff. SRÜ Anwendung fänden. Dessen Ressourcen stellen das gemeinsame Erbe der Menschheit (,common heritage of mankind“) dar und sind internationalisiert (Vöneky/Höfelmeier 2017a, Rdn 1-24; Vöneky/Höfelmeier 2017b, Rdn 1-14; Vöneky/Höfelmeier 2017c, Rdn 1-12; Vöneky/Höfelmeier 2017d, Rdn 1-9). Die Aneignung oder sonstige Eigentumsbegründung an diesen Ressourcen ist ausgeschlossen (Art. 137 SRÜ). Jede Nutzung der Ressourcen im Gebiet muss vielmehr zum Vorteil der gesamten Menschheit erfolgen (Art. 140 SRÜ) und darf ausschließlich friedlichen Zielen dienen (Art. 141 SRÜ).

Gegen eine Anwendung der Art. $133 \mathrm{ff}$. SRÜ spricht jedoch der eindeutige Wortlaut des Vertragstextes, wonach die internationalisierten Ressourcen nur mineralische Ressourcen sind (Art. 133 SRÜ). Ferner stehen dem die spätere entgegenstehende Übung der Vertragsparteien sowie die vorbereitenden Arbeiten und die Umstände des Vertragsschlusses entgegen (vgl. Wolfrum/Matz 2000, S. 455). Teil 11 (XI) SRÜ ist danach allein auf eine Regulierung der Bewirtschaftung mineralischer Ressourcen im Gebiet angelegt. Nur diese sind dem Prinzip des gemeinsamen Erbes der Menschheit unterworfen, so dass die für das Bioprospecting relevanten genetischen Ressourcen davon gerade nicht umfasst werden (Leary 2010, S. 362; Greiber 2011, S. 12; Annweiler 2017, S. 82; Leary 2004; Matz 2002, S. 289).

Auch die Internationale Meeresbodenbehörde (International Seabed Authority, ISA) hat keine Aufgaben und Befugnisse hinsichtlich des Gebietes, die auch genetische Ressourcen betreffen würden (Teil 11 (XI), Art. 157 SRÜ) (s. Wolfrum/ Matz 2000, S. 455; Proelß 2008, S. 429; Annweiler 2017, S. 84 mit weiteren Nachweisen). Das Verlangen der Entwicklungsländer nach einem einheitlichen Regime - und damit eine auf mineralische und lebende Ressourcen bezogene Befugnis - konnte sich gerade nicht durchsetzen (Guntrip 2003). Alle Aufgaben der ISA sind auf die mineralischen Ressourcen im Gebiet beschränkt (Art. 1 Abs. 1 Nr. 3, Art. 133 lit. a SRÜ).

Es bleibt daher bei dem Ergebnis, dass nach gegenwärtigem Seevölkerrecht ein freier Zugang aller Staaten zu genetischen Ressourcen jenseits nationaler Jurisdiktion gegeben ist, der praktisch aber nur von wenigen Staaten genutzt wer- 
den kann. Die faktische Verantwortung für die Bewahrung und nachhaltige Nutzung der genetischen Ressourcen liegt damit auch nur bei diesen Staaten.

Angesichts dieser Anwendung des Seerechtsübereinkommens auf das Bioprospecting verwundert es nicht, dass Staaten nach Lösungen suchen, um die mit dem Bioprospecting verbundenen Gerechtigkeitsfragen bei der Erkundung mariner genetischer Ressourcen neu zu beantworten. 2004 hat auch die Generalversammlung der Vereinten Nationen beschlossen, eine Arbeitsgruppe zu Fragen der Erhaltung und nachhaltigen Nutzung der biologischen Vielfalt der Meere in Gebieten außerhalb nationaler Gerichtsbarkeit ${ }^{4}$ einzurichten (UNGA Res. A/RES/ 59/24, Rdn 73). Trotz der besonderen Schwierigkeiten, die sich in den letzten Jahren für die multilaterale internationale Ordnung der Nachkriegszeit stellen, legte die Generalversammlung auf Empfehlung der Arbeitsgruppe (UN Doc. A/69/ 780, Annex, Rdn 1(e)) 2015 in der Res. 69/292 nieder, dass ein völkerrechtlich verbindliches Instrument unter dem Seerechtsübereinkommen der Vereinten Nationen über die Bewahrung und nachhaltige Nutzung meeresbiologischer Diversität von Gebieten jenseits nationaler Jurisdiktion entwickelt werden soll (UNGA Res. A/RES/59/24). Das Vorbereitungskomitee (Preparatory Committee, PrepCom) für dieses Übereinkommen nahm 2016 seine Arbeit auf. Gegenstand war auszuloten, wie u.a. die Bewahrung und nachhaltige Nutzung maritimer Biodiversität ,auch bezüglich der genetischen Ressourcen des Meeres, unter Einbeziehung von Fragen über die Verteilung von Vorteilen“ als Ziele erreicht werden können. Dieses Komitee tagte 2016 und 2017, bevor es unter seinem Mandat der Generalversammlung seinen Bericht mit inhaltlichen Empfehlungen für ein internationales rechtsverbindliches Instrument im Rahmen des Seerechtsübereinkommens vorlegte (UNGA Res. A/RES/69/292, Rdn 1(a); UN Doc. A/ AC.287/2017/PC.4/2, Rdn 38). Abschnitt A enthält eine nicht abschließende Liste von Elementen, denen die meisten Delegationen zugestimmt haben. Es wird u.a. der geographische Anwendungsbereich definiert; dies sind Gebiete jenseits nationaler Jurisdiktion. Gleichzeitig werden die Jurisdiktion und die bestehenden Rechte der Küstenstaaten mit Blick auf den Festlandsockel sowie die AWZ betont. Zum inhaltlichen Anwendungsbereich soll die Erhaltung und nachhaltige Nutzung mariner biologischer Diversität zählen, insbesondere mariner genetischer Ressourcen einschließlich der Frage geteilter Vorteile. Bei den generellen Prinzipien, die enthalten sein könnten, finden sich viele bekannte, wie das der Nutzung allein für friedliche Zwecke, aber auch noch widerstreitende Positionen, wie ei-

4 Vgl. Intergovernmental Conference on an International Legally Binding Instrument under the United Nations Convention on the Law of the Sea on the Conservation and Sustainable Use of Marine Biological Diversity of Areas Beyond National Jurisdiction, BBNJ. 
nerseits das Vorsorgeprinzip („precautionary approach“) und andererseits der sog. Science-based Approach, der für einen Regulierungsauftrag den wissenschaftlichen Nachweis einer Gefahr voraussetzt.

Bezüglich der marinen genetischen Ressourcen ist der geographische und materielle Anwendungsbereich jedoch umstritten. Allgemein sollen Zugang und Vorteilsausgleich geregelt werden. Ziel des Vorteilsausgleichs soll das sog. Capacity Building sich entwickelnder Staaten sein, um ihnen Zugang zu und Nutzung von genetischen Ressourcen zu ermöglichen. Zudem dient dies dem Nutzen für gegenwärtige und zukünftige Generationen und der Unterstützung von Meeresforschung.

Zusammenfassend heißt es in Teil B, der die umstrittenen Empfehlungen umfasst und zentrale Fragen als streitig aufführt: „With regard to common heritage of mankind, freedom of high seas: further discussions are required" (UN Doc. A/AC.287/2017/PC.4/2, Rdn 38). Gerade in Bezug auf marine genetische Ressourcen seien weitere Diskussionen vor allem dahingehend erforderlich,

- ob der Vertrag den Zugang zu diesen Ressourcen regeln solle,

- welche Arten von Ressourcen erfasst werden sollen,

- welche Vorteile geteilt werden sollen,

- ob Rechte am geistigen Eigentum behandelt werden sollen und

- ob es ein Monitoring für die Nutzung der marinen genetischen Ressourcen geben soll.

Ein internationaler Vertrag, der auch die nachhaltige Nutzung mariner genetischer Ressourcen jenseits der Grenzen nationaler Jurisdiktion regelt, ist damit hochumstritten. Dies betrifft insbesondere Zugangs- und Vorteilsausgleichsregeln, die nach einer Ansicht zwischen Entwicklungs- und Industrieländern vereinbart werden sollten, um die legitimatorischen Lücken des Seerechtsübereinkommens zu schließen.

Richtig ist, dass Legitimationsdefizite behoben werden müssen, die entstehen, wenn nur wenige Staaten die Technologie besitzen, genetische Ressourcen in hoheitsfreien Räumen zu nutzen. Allerdings sollte ein weiteres Auseinanderdriften der internationalen Ordnung vermieden werden. Bereits heute sind die USA nicht an die Regeln des Seerechtsübereinkommens gebunden, sofern diese nicht auch gewohnheitsrechtlich gelten. Gerade das Prinzip des gemeinsamen Erbes der Menschheit in Bezug auf mineralische Ressourcen ist und war ein Grund für die Zurückhaltung der USA bei der Ratifikation des Seerechtsübereinkommens. Einer Anwendung dieses Prinzips auf marine genetische Ressourcen widerspre- 
chen die USA zudem ausdrücklich, ${ }^{5}$ während andere Staaten es gerade als Ausgangspunkt der Verhandlungen sehen. ${ }^{6}$ Kaum lösbare Probleme ergeben sich daher, wenn das Prinzip des gemeinsamen Erbes der Menschheit die wesentliche Grundlage für neue Normen des Seevölkerrechts sein soll. Dieses Prinzip erscheint zu sehr mit den bestehenden Normen für den Abbau mineralischer Ressourcen im Seerechtsübereinkommen verbunden und durch dieses „alte“ internationalisierte Regime geprägt $\mathrm{zu}$ sein. $\mathrm{Zu}$ weitgehende Forderungen mancher Staaten könnten gerade kontraproduktiv sein, um ein tragfähiges zukünftiges Bioprospecting-Regime für das 21. Jahrhundert zu erreichen.

Optimistisch sollte stimmen, dass die Staaten das Gerechtigkeits- und Legitimationsproblem nicht nur anerkennen, sondern darum ringen, es zu lösen: Im April 2018 wurde auf einer Regierungskonferenz ein Plan für die nächsten zwei Jahre erstellt (Lajčák 2018). Die erste Sitzung der Regierungskonferenz zur Erhaltung und nachhaltigen Nutzung der biologischen Vielfalt der Meere in Gebieten außerhalb nationaler Gerichtsbarkeit fand im September 2018 statt. $^{7}$ Es wird also nach internationalen Lösungen gesucht, auch wenn das Ergebnis in weiter Ferne liegt. Dies mag manchen Beobachtern als zu wenig erscheinen, will man eine neue Technik regulieren und Legitimationslücken schließen. Dennoch ist es ein erster Schritt hin zu einer internationalen multilateralen Lösung. Gerade heute ist auch das keine Selbstverständlichkeit mehr.

\section{Bibliographie}

Abida, Heni et al. (2013): „Bioprospecting Marine Plankton“. In: Marine Drugs 11,

S. $4594-4611$.

Annweiler, Matthias (2017): Die Bewirtschaftung der genetischen Ressourcen des

Meeresbodens jenseits der Grenzen nationaler Hoheitsgewalt. Berlin: Duncker \& Humblot. Arico, Salvatore/Salpin, Charlotte (2005): Bioprospecting of Genetic Resources in the Deep

Seabed: Scientific, Legal and Policy Aspects. Yokohama: United Nations University Press.

5 Vgl. Views Expressed by the United States Delegation Related to Certain Key Issues Under Discussion at the Second Session of the Preparatory Committee on the Development of an International Legally Binding Instrument under the United Nations Convention on the Law of the Sea on the Conservation and Sustainable Use of Marine Biological Diversity 2016

6 S. auch Statement on Behalf of the Group of 77 and China at the Third PrepCom Meeting on Conservation and Sustainable Use of Marine Biological Diversity of Areas Beyond National Jurisdiction 2017

7 First session of the Intergovernmental Conference on an international legally binding instrument under the United Nations Convention on the Law of the Sea on the conservation and sustainable use of marine biological diversity of areas beyond national jurisdiction. http://www.un. org/depts/los/reference_files/calendar_of_meetings.htm, abgerufen am 04.12.2018. 
Boetius, Antje/Haeckel, Matthias (2018): „Mind the Seafloor“. In: Science 359. Nr. 6371, S. $34-36$.

Greiber, Thomas (2011): Access and Benefit Sharing in Relation to Marine Genetic Resources from Areas Beyond National Jurisdiction, A Possible Way Forward. Bonn: Bundesamt für Naturschutz.

Guntrip, Edward (2003): „The Common Heritage of Mankind: An Adequate Regime for Managing the Deep Seabed?“. In: Melbourne Journal of International Law 2, S. 376-405.

Lajčák, Miroslav (2018): „Statement by H.E. Mr. Miroslav Lajčák, President of the 72nd Session of the UN General Assembly“. In: Organizational Meeting of Intergovernmental Conference on an International Legally Binding Instrument under the United Nations Convention on the Law of the Sea on the Conservation and Sustainable Use of Marine Biological Diversity of Areas beyond National Jurisdiction of the Intergovernmental Conference (BBNJ), UNO, New York. https://www.un.org/pga/72/2018/04/16/bbnj/, abgerufen am 04.12.2018.

Leary, David K. (2004): „Bioprospecting and the Genetic Resources of Hydrothermal Vents on the High Seas: What Is the Existing Legal Position, Where Are We Heading and What Are Our Options?“. In: Macquarie Journal of International and Comparative Environmental Law 7, S. $137-178$.

Leary, David K. (2010): „International Law and the Genetic Resources of the Deep Sea“. In: Davor Vidas (Hrsg.): Law, Technology and Science for Oceans in Globalisation: IUU Fishing, Oil Pollution, Bioprospecting, Outer Continental Shelf. Leiden: Martinus Nijhoff, S. 353-369.

Leroux, Nicolas/Mbengue, Makane M. (2010): „Deep-Sea Marine Bioprospecting under UNCLOS and the CBD“. 6th ABLOS Conference. Monaco: International Hydrographic Organization.

Matz, Nele (2002): „Marine Biological Resources - Some Reflections on Concepts for the Protection and Sustainable Use of Biological Resources in the Deep Sea“. In: Non-State Actors and International Law 2. Nr. 3, S. 279-330.

Matz-Lück, Nele (2017): „Art. 241 UNLCOS“. In: Alexander Proelß (Hrsg.): United Nations Convention on the Law of the Sea (UNCLOS) - Commentary. München: Beck, S. $1624-1630$.

Mossop, Joanna (2010): „Regulating Uses of Marine Biodiversity on the Outer Continental Shelf“. In: Davor Vidas (Hrsg.): Law, Technology and Science for Oceans in Globalisation: IUU Fishing, Oil Pollution, Bioprospecting, Outer Continental Shelf. Leiden: Martinus Nijhoff, S. 319-337.

Mossop, Joanna (2015): „Marine Bioprospecting“. In: Donald R. Rothwell/Alex 0. Elferink/Karen N. Scott/Tim Stephens (Hrsg.): Oxford Handbook of the Law of the Sea. Oxford: Oxford University Press, S. 824-842.

Overmann, Jörg/Smith, David (2017): „Contributions of Microbial Resource Centers to Bioprospecting of Bacteria and Filamentous Microfungi“. In: Russell Paterson/Nelson Lima (Hrsg.): Bioprospecting - Success, Potential and Constraints. Luxemburg: Springer, S. 51-79.

Proelß, Alexander (2007): „Die Bewirtschaftung der genetischen Ressourcen des Tiefseebodens - Ein neues Seerechtsproblem?“. In: Natur und Recht 29, S. 650-656.

Proelß, Alexander (2008): „Marine Genetic Resources under UNCLOS and the CBD“. In: GYIL 51, S. $417-446$. 
Proelß, Alexander (2017): „Art. 56 UNCLOS“. In: Alexander Proelß (Hrsg.): United Nations Convention on the Law of the Sea (UNCLOS) - Commentary. München: Beck, S. 418-437.

Rayfuse, Rosemary (2017): „Art. 118 UNCLOS“. In: Alexander Proelß (Hrsg.): United Nations Convention on the Law of the Sea (UNCLOS) - Commentary. München: Beck, S. 817-830.

Scovazzi, Tullio (2011): „The Conservation and Sustainable Use of Marine Biodiversity, Including Genetic Resources, in Areas Beyond National Jurisdiction: A Legal Perspective“. http://www.un.org/Depts/los/consultative_process/ICP12_Presentations/Scovazzi_ Presentation.pdf, abgerufen am 04.12.2018.

Snelgrove, Paul (2016): „An Ocean of Discovery: Biodiversity Beyond the Census of Marine Life“. In: Planta Medica 82, S. 790-799.

Statement on Behalf of the Group of 77 and China at the Third PrepCom Meeting on Conservation and Sustainable Use of Marine Biological Diversity of Areas Beyond National Jurisdiction (2017): New York, http://www.g77.org/statement/getstatement.php? id=170327, abgerufen am 04.12.2018.

Synnes, Marianne (2007): „Bioprospecting of Organisms from the Deep Sea: Scientific and Environmental Aspects“. In: Clean Technologies and Environmental Policy 9, S. 53-59.

Views Expressed by the United States Delegation Related to Certain Key Issues Under Discussion at the Second Session of the Preparatory Committee on the Development of an International Legally Binding Instrument under the United Nations Convention on the Law of the Sea on the Conservation and Sustainable Use of Marine Biological Diversity (2016): New York, http://www.un.org/depts/los/biodiversity/prepcom_files/USA_Submission_of_ Views_Expressed.pdf, abgerufen am 04.12.2018.

Voeneky, Silja (2018): „Human Rights and Legitimate Governance of Existential and Global Catastrophic Risks“. In: Silja Voeneky/Gerald Neuman (Hrsg.): Human Rights, Democracy, and Legitimacy in a World of Disorder. Cambridge: Cambridge University Press, S. $139-162$.

Vöneky, Silja/Beck, Felix (2017): „Art. 143 UNCLOS“. In: Alexander Proelß (Hrsg.): United Nations Convention on the Law of the Sea (UNCLOS) - Commentary. München: Beck, S. $989-1001$.

Vöneky, Silja/Höfelmeier, Anja (2017a): „Art. 136 UNCLOS“. In: Alexander Proelß (Hrsg.): United Nations Convention on the Law of the Sea (UNCLOS) - Commentary. München: Beck, S. 949-957.

Vöneky, Silja/Höfelmeier, Anja (2017b): „Art. 137 UNCLOS“. In: Alexander Proelß (Hrsg.): United Nations Convention on the Law of the Sea (UNCLOS) - Commentary. München: Beck, S. $957-964$.

Vöneky, Silja/Höfelmeier, Anja (2017c): „Art. 140 UNCLOS“. In: Alexander Proelß (Hrsg.): United Nations Convention on the Law of the Sea (UNCLOS) - Commentary. München: Beck, S. $976-981$.

Vöneky, Silja/Höfelmeier, Anja (2017d): „Art. 141 UNCLOS“. In: Alexander Proelß (Hrsg.): United Nations Convention on the Law of the Sea (UNCLOS) - Commentary. München: Beck, S. $982-986$.

Wolf, Sarah (2012): „Territorial Sea“. In: Rüdiger Wolfrum (Hrsg.): Max Planck Encyclopedia of Public International Law (MPEPIL). Bd. 9. Oxford: Oxford University Press, S. 871-884.

Wolfrum, Rüdiger/Matz, Nele (2000): „The Interplay of the United Nations Convention on the Law of the Sea and the United Nations Convention on Biological Diversity“. In: Max Planck UNYB 4, S. 445-480. 